\title{
Em Busca do Orientador de Formação Ideal
}

\author{
Looking for the Perfect Mentor
}

\author{
Ana PINHEIRO SÁ凶\1, Cristina TEIXEIRA-PINTO², Rafaela VERÍSSIMO³ , Andreia VILAS-BOAS ${ }^{4}$, João FIRMINO- \\ MACHADO 5 \\ Acta Med Port 2015 Jul-Aug;28(4):486-493
}

\section{RESUMO}

Introdução: A formação médica pós-graduada consiste num processo complexo no qual os orientadores assumem um papel fundamental. Apesar da sua importância, pouco se sabe sobre os orientadores dos internos portugueses. O presente estudo pretende caracterizar os orientadores de formação em Medicina Interna em Portugal.

Material e Métodos: Realizado um estudo observacional, transversal e de carácter analítico, através um questionário online anónimo onde foram avaliadas as características demográficas dos orientadores, o seu percurso na Medicina Interna e envolvimento com o processo formativo.

Resultados: Dos 213 questionários válidos (taxa de resposta estimada de 28,4\%), a média global da satisfação com o orientador encontra-se nos 4,52 pontos ( $\pm 1,33$ ), sendo a relação entre interno e orientador classificada nos $4,86 \pm 1,04$ pontos. O orientador 'ideal' foi caracterizado como dedicado e responsável (4,9 $\pm 1,37$ pontos), com domínio de competências práticas (4,8 $\pm 1,12$ pontos) e teóricas $(4,8 \pm 1,07$ pontos). Foram identificados como preditores da satisfação dos internos com o orientador a relação estabelecida entre ambos, o envolvimento do orientador na formação, o seu dinamismo, inovação e disponibilidade [modelo explicativo de $82,5 \%$ da satisfação $\left.\left(R^{2}=0,83 ; R^{2}=0,82\right)\right]$.

Discussão: O orientador desempenha um papel preponderante no sucesso da formação pós-graduada. Os internos de Medicina Interna em Portugal encontram-se globalmente satisfeitos com os seus orientadores e valorizam preferencialmente as suas capacidades pedagógicas.

Conclusão: Este estudo aponta para a importância do orientador de formação na satisfação do interno com o seu internato, alertando para a necessidade de investir nos orientadores como forma de investimento na formação médica pós-graduada.

Palavras-chave: Educação Médica Contínua; Ensino Médico Graduado; Internato; Medicina Interna/ensino, Mentores; Portugal; Questionários.

\section{ABSTRACT}

Introduction: The authors established the profile of the Internal Medicine clinical teachers in Portugal aiming to define a future interventional strategy plan as adequate as possible to the target group and to the problems identified by the residents.

Material and Methods: Observational, transversal, analytic study. An online anonymous questionnaire was defined, evaluating the demographic characteristics of the clinical teachers, their path in Internal Medicine and their involvement in the residents learning process.

Results: We collected 213 valid questionnaires, making for an estimated response rate of $28.4 \%$. Median global satisfaction with the clinical teacher was 4.52 ( \pm 1.33 points) and the classification of the relationship between resident and clinical teacher was $4.86 \pm 1.04$ points. The perfect clinical teacher is defined by high standards of dedication and responsibility $(4.9 \pm 1.37$ points), practical ( $4.8 \pm 1.12$ points $)$ and theoretical skills ( $4.8 \pm 1.07$ points). The multiple linear regression model allowed to determine predictors of the resident's satisfaction with their clinical teacher, justifying $82,5 \%$ of the variation of satisfaction with the clinical teacher $\left(R^{2}=0.83 ; R_{a}^{2}=0.82\right)$.

Discussion: Postgraduate medical education consists of an interaction between several areas of knowledge and intervening variables in the learning process having the clinical teacher in the central role. Overall, the pedagogical abilities were the most valued by the Internal Medicine residents regarding their clinical teacher, as determinants of a quality residentship.

Conclusion: This study demonstrates the critical relevance of the clinical teacher in the satisfaction of residents with their residentship. The established multiple linear regression model highlights the impact of the clinical and pedagogical relantionship with the clinical teacher in a relevant increase in the satisfaction with the latter.

Keywords: Education, Medical, Continuing; Education, Medical, Graduate; Internal Medicine/education; Internship and Residency; Mentors; Portugal; Questionnaires.

\section{INTRODUÇÃO}

O impacto do orientador no processo de formação de futuros especialistas aptos e capacitados a dar resposta às elevadas exigências da Medicina Interna é inquestionável.

Reconhecendo o seu papel central no sucesso do internato em Medicina Interna, a necessidade de estabelecer critérios de qualidade e formação contínua dos mesmos

1. Departamento de Medicina Interna. Hospital de Santa Maria. Centro Hospitalar Lisboa Norte. Lisboa. Portugal.

2. Departamento de Medicina Interna. Unidade de Faro. Centro Hospitalar do Algarve. Faro. Portugal.

3. Departamento de Medicina Interna. Hospital de Gaia. Centro Hospitalar Vila Nova de Gaia/Espinho. Vila Nova de Gaia. Portugal.

4. Departamento de Medicina Interna. Unidade Local de Saúde de Matosinhos. Hospital Pedro Hispano. Matosinhos. Portugal.

5. Departamento de Saúde Pública. Unidade de Saúde Pública Porto Ocidental. Porto. Portugal.

$\triangle$ Autor correspondente: Ana Pinheiro Sá. ana.maria.pinheiro.sa@gmail.com

Recebido: 05 de Janeiro de 2015 - Aceite: 18 de Junho de 2015 | Copyright @ Ordem dos Médicos 2015 
tem estado na ordem do dia. Têm sido frequentemente debatidos assuntos como a importância de proporcionar formação complementar com vista a melhorar o desempenho dos formadores, criar os incentivos adequados para mantê-los motivados e estabelecer mecanismos de avaliação das suas capacidades formativas. Neste sentido, a própria Organização Mundial de Saúde (OMS) em conjunto com a World Federation for Medical Education (WFME) redigiram o Global Standards for Quality Improvement in Postgraduate Medical Education (2003) exercendo um esforço para promover elevados padrões de qualidade na educação médica. ${ }^{1}$ Com o mesmo propósito surgiu a European Academy of Teachers in General Practice and Family Medicine (EURACT), uma organização criada para promover formação e informação de elevada qualidade no âmbito da Medicina Geral e Familiar nos países europeus. ${ }^{2}$

A formação médica pós-graduada consiste na etapa que se segue à formação básica na qual os jovens médicos são treinados sob supervisão até à prática autónoma e independente da Medicina. ${ }^{1}$ Este processo engloba a aquisição de diversos tipos de competências, como a aprendizagem e aplicação de conhecimentos teóricos, a prática de procedimentos médicos (invasivos ou não) e o treino de competências de comunicação interpessoal. ${ }^{1}$ A participação de médicos especialistas neste processo formativo é absolutamente indispensável, sendo parte dos seus deveres e responsabilidades cooperar de forma sinérgica e respeitosa com os internos, na orientação do diagnóstico e terapêutica, nos procedimentos médicos, nas relações com os doentes e familiares e com os demais colegas, tal como predisposto no Código Deontológico da Ordem dos Médicos. ${ }^{3}$

Ao longo do percurso pós-graduado, o orientador de formação assume um papel fundamental. Por um lado, ao desempenhar o papel de avaliador, é responsável por criar, em parceria com o interno e o serviço que integram, pontos de verificação do processo de aprendizagem que permitem um crescimento tutoreado e por etapas, baseado no estudo e experiência clínica supervisionada. ${ }^{4}$ Por outro lado, o vínculo criado entre o interno e o seu orientador afigura-se fundamental para a aprendizagem por role modelling. ${ }^{5}$ A aprendizagem por modelos ou mentores é uma poderosa ferramenta de ensino, nem sempre usada de forma consciente, que permite transmitir conhecimentos, competências e valores médicos de forma muito eficiente. ${ }^{5}$ Aprender por role modelling implica a observação e a reflexão num processo complexo em parte consciente e noutra inconsciente. ${ }^{5,6} \mathrm{Na}$ verdade, vários estudos têm demonstrado que a aprendizagem através de modelos terá provavelmente um maior impacto na formação que as restantes formas de aprendizagem. ${ }^{7,8}$ Tendo em consideração que uma parte significativa do processo formativo de futuros médicos depende de processos não completamente conscientes, compreende-se a importância de incentivar o treino dos clínicos para se tornarem orientadores cada vez mais capacitados.

A formação dos nossos formadores e definição de competências chave para o orientador ideal constituem portanto assuntos da maior relevância na formação médica pós- -graduada. No entanto, muito pouco é conhecido acerca da realidade dos orientadores de formação em Portugal e das perspetivas dos internos acerca dos mesmos.

No caso da Medicina Interna, a participação dos mentores afigura-se particularmente importante tendo em consideração o seu âmbito abrangente e visão integradora da constelação de características fisiopatológicas do doente que implicam também uma grande interação com outras especialidades. ${ }^{9}$ Importa realçar que a Medicina Interna constitui a base da dinâmica e organização hospitalar, razão pela qual nos últimos anos cerca de 13 a $15 \%$ do total de vagas de internato de formação específica têm sido dedicados a essa especialidade. ${ }^{10}$

Salientamos ainda que, até ao momento, não existe nenhum estudo nacional que avalie a satisfação dos internos de Medicina Interna com o seu orientador de formação, havendo somente artigos de outras especialidades., ,11-14 $^{-14}$ Internacionalmente multiplicam-se os estudos de opinião de internos acerca da qualidade da sua formação pós-graduada, procurando-se uma adequada ferramenta de medida dessa mesma qualidade. O questionário Postgraduate Hospital Education Environment Measure (PHEEM), validado em múltiplos países, ${ }^{15-19}$ parece ser o instrumento mais consensual. Trata-se de um questionário de 40 perguntas que caracteriza três domínios, nomeadamente as percepções de autonomia, de qualidade do ensino e suporte social. Um dos elementos identificados como mais relevante é o papel do orientador. ${ }^{20}$

Numa altura em que o número de vagas de internato de formação específica em Medicina Interna aumenta ano após ano e perante a vontade de internos e orientadores de garantir um internato de qualidade, os autores propuseram-se a caracterizar os orientadores de formação em Medicina Interna em Portugal com vista a permitir um planeamento de áreas e estratégias de intervenção o mais adequado possível ao grupo alvo e às carências detetadas pelos internos.

\section{MATERIAL E MÉTODOS}

Desenvolveu-se um estudo observacional, transversal e carácter analítico. Foi criado um questionário online anónimo utilizando a plataforma Survio ${ }^{\mathrm{TM}}$ onde foram avaliadas as características demográficas dos orientadores de formação, o seu percurso na Medicina Interna (incluindo tempo de atividade assistencial e formação pós-graduada adicional) e o seu envolvimento com o processo formativo do interno.

Os itens do questionário foram definidos por um focus group de elementos do núcleo de internos da Sociedade Portuguesa de Medicina Interna, com representação de várias zonas do país e com diferentes vivências formativas, baseando as opiniões na pesquisa bibliográfica prévia e nas suas experiências com a formação.

O questionário é composto por 45 itens cujas respostas foram quantificadas através de uma escala de Likert com seis níveis, tal como se pode constatar na Tabela 1. Os itens foram dicotomizados em respostas positivas (4-6) 
Tabela 1 - Questões selecionadas

Quão satisfeito estás com o teu orientador?
Como classificas a tua relação com o teu orientador?
Como te foi atribuído o teu orientador:
Escolha própria?
Escolha da direção de serviço?
O teu orientador assegura-se de que cumpres os objetivos do teu
Como classificarias o teu orientador em relação a:
Competências teóricas
Competências práticas
Capacidade de comunicação com os internos
Capacidade de comunicação interpares
Experiência de ensino
Produção científica
Conhecimento do programa e grelha de avaliação o internato
Dinamismo e inovação
Dedicação e responsabilidade
Disponibilidade

Como classificarias o envolvimento do teu orientador no teu (tua):

Planeamento de estágios

Participação em atividades docentes

Participação em trabalhos de investigação

Participação em protocolos

Discussão de casos clínicos

Formação teórica

Formação prática

Formação pós-graduada

Participação em congressos / reuniões científicas

Ordena por ordem decrescente de importância as características que, para ti, deveria ter o orientador ideal:

Ser empático

Ser didático

Mostrar-se interessado

Ser motivador

Estar disponível

Dominar conhecimentos teóricos

Dominar conhecimentos práticos

Manter-se atualizado

Desafiar-te na discussão de casos clínicos

Desafiar-te para a investigação / novos projetos

Ser exigente com o teu trabalho 
ou negativas (1-3). Num universo estimado de 750 internos a nível nacional, o questionário foi divulgado via email para os internos constantes da rede de contactos do Núcleo de Internos da Sociedade Portuguesa de Medicina Interna (661 contactos) e divulgado também numa rede social, tendo ficado disponível para resposta durante todo o mês de Dezembro de 2013. Foram incluídos todos os questionários totalmente respondidos por médicos a integrar o internato de formação específica em Medicina Interna. Foram definidos como critérios de exclusão o preenchimento incompleto do questionário e o seu preenchimento por internos de outras especialidades que não Medicina Interna.

\section{Análise estatística}

Realizou-se uma análise descritiva das variáveis de caracterização dos orientadores de formação, com apresentação de frequências e percentagens para as variáveis categóricas, e médias e desvio padrão para as variáveis contínuas.

A avaliação da relação dos internos com o seu orientador e envolvimento nas tarefas pedagógicas foi realizada através da definição de um conjunto de itens, numa escala de Likert com seis níveis, tendo sido apresentado para cada item o seu respetivo valor médio e desvio-padrão. 0 teste à normal distribuição dos dados foi realizada com recurso ao teste de Shapiro-Wilk ou pela análise dos valores de assimetria e achatamento, para os casos das variáveis psicométricas (valores máximos toleráveis de assimetria e achatamento de 3 e 8 , respetivamente).

Ajustou-se um modelo de regressão linear múltipla, com método de entrada de dados stepwise, como forma de determinar os preditores da satisfação com o orientador de formação em Medicina Interna.

Todos os valores reportados de $p$ são bicaudados, com um nível de significância ( $\alpha$ ) de 0,05. A análise de dados foi

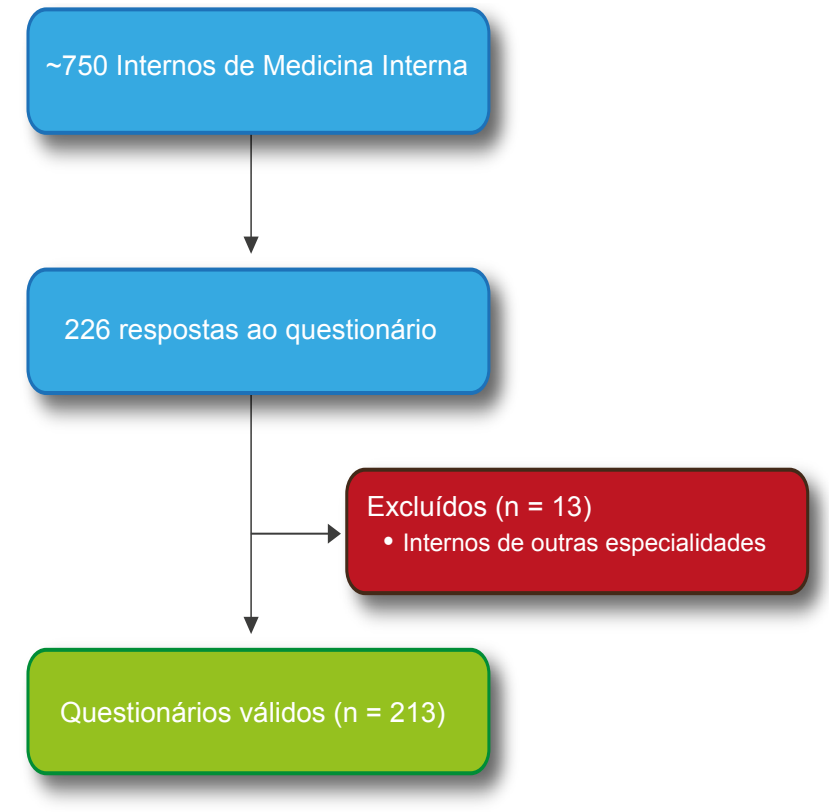

Figura 1 - Fluxograma realizada com recurso ao software SPSS, versão 22.

\section{RESULTADOS}

No período estipulado para resposta ao questionário, foram submetidos 226 formulários completos, dos quais, 13 foram excluídos por não serem internos de Medicina Interna. De um universo estimado de cerca de 750 internos de Medicina Interna em Portugal em 2013, foram obtidos 213 questionários válidos (Fig. 1), perfazendo uma taxa de resposta de $28,4 \%$, dos quais $153(71,8 \%)$ dos respondentes eram do sexo feminino e $60(28,2 \%)$ do sexo masculino, com uma idade média de $28,7( \pm 2,20)$ anos.

A Tabela 2 mostra as características socio-demográficas dos orientadores de formação de Medicina Interna em Portugal. A maioria dos orientadores de formação é do sexo feminino $(68,5 \%)$, com idade inferior a 50 anos $(67,2 \%)$, com mais de 10 anos de especialidade $(55,4 \%)$ e sem formação pós-graduada $(67,1 \%)$. O número de internos por orientador de formação é, na maioria dos casos, inferior ou igual a $2(82,6 \%)$.

Tabela 2 - Caraterização demográfica dos orientadores

\begin{tabular}{|c|c|c|}
\hline & $\mathbf{n}$ & $\%$ \\
\hline \multicolumn{3}{|l|}{ Sexo } \\
\hline Masculino & 67 & 31,5 \\
\hline Feminino & 146 & 68,5 \\
\hline \multicolumn{3}{|l|}{ Idade } \\
\hline$<40$ anos & 70 & 32,9 \\
\hline $40-50$ anos & 73 & 34,3 \\
\hline $50-60$ anos & 64 & 30,0 \\
\hline$>60$ anos & 6 & 2,8 \\
\hline \multicolumn{3}{|l|}{ Área geográfica } \\
\hline Norte & 90 & 42,3 \\
\hline Centro & 67 & 31,5 \\
\hline Sul & 53 & 24,9 \\
\hline Ilhas & 3 & 1,4 \\
\hline \multicolumn{3}{|l|}{ Anos de especialidade } \\
\hline$<2$ anos & 3 & 1,4 \\
\hline $2-5$ anos & 40 & 18,8 \\
\hline $5-10$ anos & 52 & 24,4 \\
\hline$>10$ anos & 118 & 55,4 \\
\hline \multicolumn{3}{|l|}{ Grau académico } \\
\hline Licenciado & 143 & 67,1 \\
\hline Pós-graduado & 50 & 23,5 \\
\hline Mestrado & 13 & 6,1 \\
\hline Professor Doutor & 7 & 3,3 \\
\hline \multicolumn{3}{|c|}{ Número de internos a cargo } \\
\hline 1 & 79 & 37,1 \\
\hline 2 & 97 & 45,5 \\
\hline 3 & 32 & 15,0 \\
\hline 4 & 3 & 1,4 \\
\hline NR & 2 & 0,9 \\
\hline
\end{tabular}


Características objetivadas e desejadas nos orientadores de formação

A média global da satisfação com o orientador encontra-se nos 4,52 ( $\pm 1,33$ pontos) e a classificação da relação entre o interno e o orientador nos 4,86 $\pm 1,04$ pontos.

A Tabela 3 mostra a valorização que os internos atribuem aos orientadores de formação em várias áreas de perfil pessoal e de envolvimento nas atividades do interno relacionadas com a sua formação. Da sua análise retiramos que o orientador 'tipo' de Medicina Interna apresenta como principais características elevados padrões de dedicação e responsabilidade (média de 4,9 $\pm 1,37$ pontos), competências práticas (média de 4,8 $\pm 1,12$ pontos) e teóricas (média de 4,8 $\pm 1,07$ pontos). As principais intervenções dos orientadores nas atividades dos internos são sobretudo ao nível da discussão de casos clínicos (média de 4,5 \pm 1,32 pontos), formação prática (média de $4,4 \pm 1,40$ pontos) e no planeamento dos estágios (média de $4,1 \pm 1,41$ pontos).

Foi ainda constatado que 147 (69,0\%) dos orientadores de formação asseguram o cumprimento dos objetivos do internato dos seus internos.

Quando inquiridos acerca das caraterísticas mais desejáveis num orientador ideal, a caraterística mais valorizada foi a capacidade didática, seguida da disponibilidade, da capacidade para motivar e do interesse pela formação do interno. Foram considerados como elementos de menor relevância num orientador ideal, a capacidade para estimular o desenvolvimento de projetos de investigação, a atualização contínua e o desafio para a discussão de casos clínicos.

\section{Determinação dos preditores de satisfação com o orientador de formação em Medicina Interna}

O ajustamento do modelo de regressão linear múltipla permitiu identificar como preditores do valor de satisfação dos internos com o orientador variáveis como a relação com o orientador, envolvimento do orientador na formação teórica, classificação do orientador em dinamismo e inovação, competências práticas e disponibilidade, capacidade para assegurar o cumprimento dos objetivos do internato, experiência de ensino e envolvimento no planeamento de estágios.

Tabela 3 - Competências dos orientadores (escala de Likert de 1 a 6 )

Média $( \pm \mathrm{DP})$

Classificação do orientador em:

Competências teóricas

$4,80( \pm 1,07)$

Competências práticas

$4,84( \pm 1,12)$

Comunicação com os internos

$4,76( \pm 1,15)$

Comunicação interpares

$4,61( \pm 1,11)$

Experiência de ensino

$4,28( \pm 1,24)$

Produção científica

$3,42( \pm 1,40)$

Conhecimento da grelha de avaliação do internato

$4,48( \pm 1,29)$

Dinamismo e inovação

$3,99( \pm 1,41)$

Dedicação e responsabilidade

$4,85( \pm 1,34)$

Disponibilidade

$4,71( \pm 1,37)$

Envolvimento do orientador em:

Planeamento de estágios

$4,13( \pm 1,41)$

Participação em atividades docentes

$3,43( \pm 1,57)$

Trabalhos de investigação

Elaboração de protocolos

Discussão de casos clínicos

Formação teórica

$4,10( \pm 1,43)$

Formação prática

Formação pós-graduado 
Tabela 4 - Modelo de predição da satisfação dos internos com o orientador

Impacto no Aumento na Satisfação com Orientador

$\begin{array}{lcc}\text { Variáveis Independentes } & \text { B (IC 95\%) } & p \\ \text { Relação com o orientador } & 0,45(0,35-0,55) & <0,001 \\ \text { Assegura cumprimento de objetivos do internato } & 0,31(0,10-0,52) & <0,001 \\ \text { Competências práticas } & 0,20(0,10-0,29) & <0,01 \\ \text { Envolvimento na formação teórica } & 0,14(0,05-0,23) & <0,01 \\ \text { Dinamismo e inovação } & 0,13(0,04-0,22) & <0,01 \\ \text { Experiência de ensino } & 0,13(0,04-0,22) & 0,01 \\ \text { Disponibilidade } & 0,11(0,02-0,19) & 0,01 \\ \text { Envolvimento no planeamento de estágios } & 0,11(0,02-0,19) & \end{array}$

Este modelo explica $82,5 \%$ da variação da satisfação com o orientador $\left(R^{2}=0,83 ; R_{a}^{2}=0,82\right)$, sendo a variável mais preponderante a qualidade da relação com o orientador, conforme exposto na Tabela 4 em que é visível que o aumento de 1 ponto na qualidade da relação com o orientador aumenta 0,45 pontos na satisfação com o mesmo.

\section{DISCUSSÃO}

O processo de formação pós-graduada de um médico consiste numa interação entre diversas áreas do conhecimento e variáveis intervenientes na aprendizagem no qual o papel do orientador de formação é central.

Com a rede de contatos do Núcleo de Internos da Sociedade Portuguesa de Medicina Interna foi possível informar praticamente todo o universo de internos de formação específica em Medicina Interna acerca do questionário. Obteve-se uma taxa de resposta superior a $25 \%$, considerando-se, portanto, uma taxa de resposta adequada para a metodologia adotada.

Em Portugal, os orientadores de formação em Medicina Interna são predominantemente jovens $(67,2 \%$ com idade igual ou inferior a 50 anos) e sobretudo do sexo feminino ( $n=68,5 \%$ ). Podemos especular que a elevada frequência de orientadores de idades mais jovens poderá ser explicada pela hierarquia natural vigente na maioria dos serviços hospitalares em que os elementos séniores ocupam preferencialmente lugares de chefia e gestão; com eventual maior proximidade às atividades formativas por parte dos orientadores mais jovens. Esta organização apesar de beneficiar seguramente a estrutura dos serviços pode, em consequência, privar os internos da partilha de saber e experiência que os profissionais mais experientes terão para dar, pelo que se considera importante que o envolvimento ativo dos assistentes mais graduados e diretores de serviço seja estimulado e promovido como parte integrante da formação do interno.

Constatou-se que em média cada orientador tem a seu cargo dois internos de Medicina Interna, o que se adequa às recomendações atuais, tendo em conta a disponibilida- de necessária por parte do orientador para assegurar uma formação adequada. Curiosamente, um número considerável de orientadores têm a seu cargo três ou mais internos (cerca de 17\%). Ainda que não seja possível averiguar as condições em que tal se verificou, este poderá ser o reflexo do número insuficiente de internistas vocacionados e motivados para a formação existentes em alguns serviços, para as necessidades impostas pelo crescente número de internos, sobrecarregando aqueles que se encontram mais envolvidos no projeto formativo. Nesta avaliação não foram contabilizados os internos da formação específica de outras especialidades e do ano comum, tal como alunos de Medicina que se encontram também sob a alçada destes mesmos orientadores. Esta sobrecarga de trabalho imposta pela tutoria de demasiados internos pode ser um fator de descontentamento e desincentivo à atividade pedagógica por parte dos orientadores. Desta forma, os autores consideram fundamental que se desenvolvam estratégias para envolver um maior número de internistas na atividade formativa. Além disso, num tempo em que há pressão para aumentar o número de vagas de internato de formação específica, este deverá ser um alerta para que sejam asseguradas as adequadas condições formativas dos serviços.

Os internos de Medicina Interna em Portugal encontram-se globalmente satisfeitos com os seus orientadores de formação (média de respostas 4,52 $\pm 1,33$ ), com os quais estabelecem uma relação positiva $(4,86 \pm 1,04)$. Neste estudo foi possível criar um modelo justificativo da satisfação dos internos com os seus orientadores que poderá fornecer algumas indicações sobre as áreas nas quais os orientadores poderão optimizar a sua formação. A relação formativa entre o interno e o orientador foi na verdade um dos itens mais valorizados, reforçando os dados já apresentados por outros autores que evidenciam o papel central do role modelling na formação médica. ${ }^{5,21,22}$

Lombarts et al, num estudo que abarcou internos de várias especialidades, definiram-se como principais preditores de satisfação o ambiente clínico de aprendizagem, profissionalismo face aos internos, avaliação dos conheci- 
mentos e capacidades, comunicação de objetivos e feedback, todos eles relacionados com a relação estabelecida entre orientador e interno. ${ }^{21}$

De forma global, foram as capacidades pedagógicas o mais valorizado pelos internos de Medicina Interna relativamente ao seu orientador, como determinantes de um internato de qualidade. Vieira et al chegou também à conclusão que eram os itens relacionados com a percepção de qualidade de ensino (essencialmente os ligados ao papel do orientador), os que tinham mais impacto. ${ }^{16}$

Também características como a disponibilidade e inovação do orientador, a formação teórica e experiência de ensino, as competências práticas, a capacidade para assegurar cumprimento de objetivos e o envolvimento no planeamento de estágios demonstraram um impacto significativo no aumento da satisfação com o orientador.

Os orientadores têm um papel fundamental na formação e os resultados do questionário sugerem que se encontram envolvidos e motivados para a atividade pedagógica. $\mathrm{Na}$ verdade, nos itens do questionário referentes à participação do orientador nas diversas fases do processo formativo, a média de respostas ficou sempre na faixa positiva da escala de Likert (mínimo 3,04 $\pm 1,59$ e máximo 4,49 $\pm 1,32$ ), sugerindo um envolvimento positivo dos orientadores com o internato. Por outro lado, também no que se refere à dedicação e responsabilidade $(4,85 \pm 1,34)$, disponibilidade $(4,71 \pm 1,37)$ e dinamismo e inovação $(3,99 \pm 1,41)$, os orientadores foram classificados de forma positiva, indicando também que se encontram motivados para a formação.

Os internos de Medicina Interna continuam a procurar no seu orientador alguém disponível e dedicado, didático, motivador e que se mostre interessado na formação global. É de realçar que os itens com mais baixa classificação se relacionaram com produção científica e atividades de investigação, o que, em conjunto com o nível global de formação pós-graduada dos orientadores realça a importância de maior investimento na investigação e formação contínuas. O estímulo para o desenvolvimento de projetos de investigação, a atualização científica e o desafio para a discussão de casos clínicos, não são fatores considerados como essenciais, apesar do peso que eles têm na avaliação final do internato e na formação de um bom interno de Medicina Interna. No entanto, é possível que isso se prenda com o facto que um orientador mais dedicado à formação motivará mais o interno a progredir do ponto de vista académico.

A falta de incentivos para a investigação e a pouca cultura de atualização e investimento na carreira académica em Portugal, refletem-se consequentemente nos internos e no acesso que estes têm ao meio científico. É importante notar que a criação de programas de investigação contribui significativamente para o crescimento dos serviços e aumento das oportunidades formativas, com um importante papel a ser desempenhado pelos séniores, quer como mentores, quer como agentes de mudança da organização dos serviços. ${ }^{23}$ No entanto, a produção científica nacional continua largamente dependente dos meios universitários e observam-se grandes assimetrias nacionais no investimento em investigação e na publicação científica, que necessitam ser colmatados.

Apesar da maioria dos orientadores de formação assegurar que os internos cumprem os objetivos do internato, a existência de $31 \%$ de orientadores que não o fazem é vista como possível área a optimizar, tratando-se este de um dos objetivos centrais da tutoria. Da mesma forma, a elaboração de planos individuais de internato deve ser vista como um elemento essencial, pelo que deverá ser estimulada a sua execução e revisão regulares por parte dos orientadores.

Importa salientar que este estudo tem diversas limitações, nomeadamente o facto de se basear numa amostra de conveniência, tendo em conta que a resposta ao questionário foi voluntária e terá certamente sido realizada pelos internos mais motivados para os assuntos formativos. Da mesma forma, tal como foi já referido, seria importante repetir este estudo utilizando um questionário adequadamente validado para a realidade portuguesa e que conseguisse abranger uma maior percentagem da população. Importa também salientar que a satisfação dos internos com a formação não é necessariamente sinónimo de uma formação cientificamente melhor, focando-se este estudo na mensuração de variáveis psicométricas relacionadas com satisfação e não diretamente qualidade do internato ou com a real capacitação dos internos. Como já referimos, a aprendizagem é um processo complexo no qual os mentores têm um papel relevante mas não exclusivo, e no qual factores como a motivação (também ela influenciada pela satisfação e reforço positivo dos pares), as experiências e as necessidades que advêm da prática desempenham também papéis importantes. ${ }^{24}$ Seria igualmente interessante relacionar a satisfação dos internos com os orientadores com o seu desempenho nas provas de avaliação médica, ainda que também nesse cenário a interpretação dos dados seria dificultada pelo viés inerente às atuais formas de avaliação.

O interesse pela formação médica pós-graduada consiste num tema da atualidade da comunidade científica internacional, no qual a Medicina Interna em Portugal se tem mantido pouco ativa. Este estudo constitui uma primeira visão daquelas que são consideradas as características do orientador de formação 'ideal' em Medicina Interna. Tendo em conta que uma parte significativa do processo formativo passa pela aprendizagem por modelagem, na qual os orientadores de formação são paradigma de competências, comportamentos e atitudes, este estudo pode fornecer informação valiosa na construção de um sistema de educação pós-graduada de maior qualidade.

\section{CONCLUSÃO}

Os resultados deste estudo apontam para a importância fulcral do orientador de formação na satisfação do interno com a sua formação. Do modelo justificativo que foi possível estabelecer, realça-se o impacto da relação pedagógica e clínica com o orientador num aumento significativo dos níveis de satisfação com o mesmo. Este estudo levanta 
mais questões do que respostas, pretendendo relembrar a importância de mantermos uma formação médica de qualidade e que prepare os internos de Medicina Interna para os desafios da Medicina Interna do futuro. Seria fundamental a realização de estudos mais aprofundados neste âmbito, que considerem alguns dos aspetos que não foram aqui considerados, como o real número de internos e alunos de Medicina que os orientadores de formação têm a seu cargo. A criação e desenvolvimento de estratégias para envolver mais os internistas no processo formativo poderá ser uma importante via de atuação futura.

\section{AGRADECIMENTOS}

Os autores agradecem o apoio institucional da Sociedade Portuguesa de Medicina Interna e individualmente aos seus orientadores de formação pela colaboração na execução do artigo, assim como a todos os internos que responderam ao questionário.

\section{REFERÊNCIAS}

1. Karle H; Adams O; Bajaj J; Baron-Maldonado M; Cravioto A; Dal Poz $\mathrm{M}$, et al. WHO | Postgraduate Medical Education - WFME Global Standards for Quality Improvement [Internet]. WHO. 2002 [consultado 2014 Dez 8]. Disponível em: http://www.who.int/workforcealliance/knowledge/ toolkit/45/en/.

2. Euract. [consultado 2015 Abr 9]; Disponível em: http://www.euract.eu/.

3. Código Deontológico da Ordem dos Médicos. [consultado $2015 \mathrm{Abr} 18$ ]; Disponível em: https://www.ordemdosmedicos.pt/?lop=conteudo\&op=9 c838d2e45b2ad1094d42f4ef36764f6\&id=cc42acc8ce334185e0193753 adb6cb77.

4. lobst WF, Sherbino J, Cate OT, Richardson DL, Dath D, Swing SR, et al. Competency-based medical education in postgraduate medical education. Med Teach. 2010;32:651-6.

5. Cruess SR, Cruess RL, Steinert Y. Role modelling - making the most of a powerful teaching strategy. BMJ. 2008;336:718-21.

6. Epstein RM, Cole DR, Gawinski BA, Piotrowski-Lee S, Ruddy NB How students learn from community-based preceptors. Arch Fam Med. 1998;7:149.

7. Park J, Woodrow SI, Reznick RK, Beales J, MacRae HM. Observation, reflection, and reinforcement: surgery faculty members' and residents' perceptions of how they learned professionalism: Acad Med. 2010;85:134-9.

8. Ratanawongsa N, Bolen S, Howell EE, Kern DE, Sisson SD, Larriviere D. Residents' perceptions of professionalism in training and practice: barriers, promoters, and duty hour requirements. J Gen Intern Med. 2006;21:758-63.

9. Programa de Formação em Medicina Interna. Portaria $n^{\circ} 614 / 2010$ de 3 de Agosto.

10. Mapa de vagas do concurso do Internato Médico de 2009 a 2014. [consultado 2015 Abr 18]; Disponível em http://www.acss.min-saude.pt/

11. Azevedo A, Domingues B, Moura J, Santos L. Estão os internos satisfeitos com o internato de Medicina Geral e Familiar? Rev Port Med Geral Fam. 2014;30:24-30.

12. Carvalho F, Ventura T, Barroso R. Perfil de competências do orientador de formação. Rev Port Med Geral Fam. 2004;20:147-52.

13. Vieira D, Viegas I, Furtado N. Satisfação profissional em médicos da carreira de clínica geral. Acta Med Port. 1995;8:531-5.

\section{PROTECÇÃO DE PESSOAS E ANIMAIS}

Os autores declaram que os procedimentos seguidos estavam de acordo com os regulamentos estabelecidos pelos responsáveis da Comissão de Investigação Clínica e Ética e de acordo com a Declaração de Helsínquia da Associação Médica Mundial.

\section{CONFIDENCIALIDADE DOS DADOS}

Os autores declaram ter seguido os protocolos do seu centro de trabalho acerca da publicação de dados.

\section{CONFLITOS DE INTERESSE}

Os autores declaram que não houve conflitos de interesse na realização deste trabalho.

\section{FONTES DE FINANCIAMENTO}

Este trabalho não recebeu qualquer contribuição, subsídio ou bolsa.

14. Pinto da Costa M, Guerra C, Malta R, Moura M, Carvalho S, Mendonça D. Internato de psiquiatria rumo a um futuro global: a perspectiva dos internos em Portugal. Acta Med Port. 2013;26:357-60.

15. Riquelme A, Herrera C, Aranis C, Oporto J, Padilla O. Psychometric analyses and internal consistency of the PHEEM questionnaire to measure the clinical learning environment in the clerkship of a Medical School in Chile. Med Teach. 2009;31:e221-5.

16. Vieira JE. The postgraduate hospital educational environment measure (PHEEM) questionnaire identifies quality of instruction as a key factor predicting academic achievement. Clin São Paulo Braz. 2008;63:741-6.

17. Shokoohi S, Emami AH, Mohammadi A, Ahmadi S, Mojtahedzadeh R. Psychometric properties of the Postgraduate Hospital Educational Environment Measure in an Iranian hospital setting. Med Educ Online 2014;19.

18. Al-Shiekh $\mathrm{MH}$, Ismail $\mathrm{MH}, \mathrm{Al}-\mathrm{Kh}$ ater SA. Validation of the postgraduate hospital educational environment measure at a Saudi university medical school. Saudi Med J. 2014;35:734-8.

19. Shimizu T, Tsugawa $Y$, Tanoue $Y$, Konishi R, Nishizaki $Y$, Kishimoto M, et al. The hospital educational environment and performance of residents in the General Medicine In-Training Examination: a multicenter study in Japan. Int J Gen Med. 2013;6:637-40

20. Boor K, Scheele F, van der Vleuten CP, Scherpbier AJ, Teunissen PW, Sijtsma K. Psychometric properties of an instrument to measure the clinical learning environment. Med Educ. 2007;41:92-9.

21. Lombarts KM, Heineman MJ, Arah OA. Good clinical teachers likely to be specialist role models: results from a multicenter cross-sectional survey. PloS One. 2010;5:e15202.

22. Yedidia MJ, Schwartz MD, Hirschkorn C, Lipkin M. Learners as teachers: the conflicting roles of medical residents. J Gen Intern Med. 1995;10:615-23.

23. Rothberg MB, Kleppel R, Friderici JL, Hinchey K. Implementing a resident research program to overcome barriers to resident research. Acad Med J Assoc Am Med Coll. 2014;89:1133-9.

24. Taylor DC, Hamdy $\mathrm{H}$. Adult learning theories: implications for learning and teaching in medical education: AMEE Guide No. 83. Med Teach. 2013;35:e1561-72. 
Ana PINHEIRO SÁ, Cristina TEIXEIRA-PINTO, Rafaela VERÍSSIMO, Andreia VILAS-BOAS, Firmino MACHADO

\section{Em Busca do Orientador de Formação Ideal}

Acta Med Port 2015:28:486-493

Publicado pela Acta Médica Portuguesa, a Revista Científica da Ordem dos Médicos

Av. Almirante Gago Coutinho, 151

1749-084 Lisboa, Portugal.

Tel: +351218428 215

E-mail: submissao@actamedicaportuguesa.com

www.actamedicaportuguesa.com

ISSN:0870-399X | e-ISSN: 1646-0758

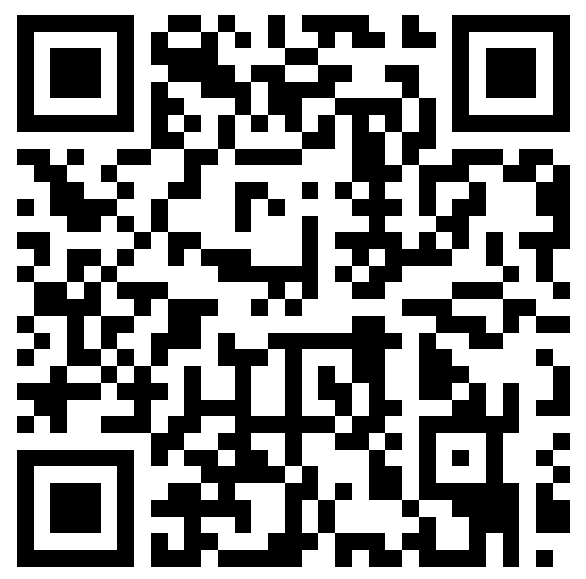

